\title{
Delayed onset of hyperglycaemia in a mouse model with impaired glucagon secretion demonstrates that dysregulated glucagon secretion promotes hyperglycaemia and type 2 diabetes
}

\author{
N. Gustavsson • T. Seah • Y. Lao • G. K. Radda • \\ T. C. Südhof • W. Han
}

Received: 16 September 2010 / Accepted: 20 September 2010 /Published online: 27 October 2010

(C) Springer-Verlag 2010

\begin{abstract}
Aims/hypothesis Type 2 diabetes is caused by relative deficiency of insulin secretion and is associated with dysregulation of glucagon secretion during the late stage of diabetes development. Like insulin secretion from beta cells, glucagon secretion is dependent on calcium signals and a calcium sensing protein, synaptotagmin-7. In this study, we tested the relative contribution of dysregulated glucagon secretion and reduced insulin release in the development of hyperglycaemia and type 2 diabetes by using synaptotagmin-7 knockout (KO) mice, which exhibit glucose intolerance, reduced insulin secretion and nearly abolished $\mathrm{Ca}^{2+}$-stimulated glucagon secretion.

Methods We fed the synaptotagmin-7 KO and control mice with a high-fat diet (HFD) for 14 weeks, and compared
\end{abstract}

N. Gustavsson · T. Seah · G. K. Radda • W. Han

Laboratory of Metabolic Medicine,

Singapore Bioimaging Consortium, A*STAR,

Singapore, Republic of Singapore

Y. Lao $\cdot$ T. C. Südhof

Department of Molecular and Cellular Physiology,

Stanford University School of Medicine,

Palo Alto, CA, USA

W. Han

Department of Biochemistry, Yong Loo Lin School of Medicine,

National University of Singapore,

Singapore, Republic of Singapore

W. Han $(\bowtie)$

\#02-02 Helios, 11 Biopolis Way,

Singapore 138667, Republic of Singapore

e-mail: weiping_han@sbic.a-star.edu.sg their body weight, glucose levels, glucose and insulin tolerance, and insulin and glucagon secretion.

Results On the HFD, synaptotagmin-7 KO mice showed progressive impairment of glucose tolerance and insulin secretion, along with continued maintenance of a low glucagon level. The control mice were less affected in terms of glucose intolerance, and showed enhanced insulin secretion with a concurrent increase in glucagon levels. Unexpectedly, after 14 weeks of HFD feeding, only the control mice displayed resting hyperglycaemia, whereas in synaptotagmin-7 KO mice defective insulin secretion and reduced insulin sensitivity were not sufficient to cause hyperglycaemia in the absence of enhanced glucagon secretion.

Conclusions/interpretation Our data uncover a previously overlooked role of dysregulated glucagon secretion in promoting hyperglycaemia and the ensuing diabetes, and strongly suggest maintenance of adequate regulation of glucagon secretion as an important therapeutic target in addition to the preservation of beta cell function and mass in the prevention and treatment of diabetes.

Keywords Exocytosis - Glucose tolerance test - Hepatic glucose production - Insulin resistance - Insulin secretion . Insulin tolerance test $\cdot$ Knockout mice $\cdot$ Pancreatic islets . Pyruvate tolerance test
Abbreviations
GTT Glucose tolerance test
HFD High-fat diet
ITT Insulin tolerance test
KRH Krebs-Ringer-HEPES
KO Knockout
LFD Low-fat diet 


\section{Introduction}

Glucose homeostasis is maintained by two counteracting hormones from the pancreas: insulin from beta cells and glucagon from alpha cells [1]. Under normal conditions, insulin is released when blood glucose is high to stimulate glucose uptake and to inhibit glucose production, while low glucose stimulates glucagon secretion, which acts on the liver to promote glycogenolysis and gluconeogenesis [1, 2]. Insulin and glucagon levels are tightly regulated to achieve a proper balance for the maintenance of glucose homeostasis. When the balance of these two hormones is disrupted, proper glucose levels cannot be maintained.

Type 2 diabetes is the result of relative insulin deficiency, i.e. impaired insulin secretion due to beta cell dysfunction and/or reduced beta cell mass that fail to overcome peripheral insulin insensitivity [3-5]. Consequently, most previous studies on the pathophysiology of type 2 diabetes were mainly focused on the interplay between beta cell dysfunction and insulin resistance [6]. Although dysregulated glucagon secretion and elevated glucagon levels are known to be associated with high blood glucose in type 2 diabetes [7, 8], these conditions are usually considered a consequence [9] instead of a cause [10] of diabetes, and it remains controversial whether glucagon, particularly dysregulated oversupply of glucagon, plays an active role in diabetes development [2].

Exocytosis of both insulin and glucagon granules is a calcium-dependent process, and is regulated by the same calcium sensor, synaptotagmin-7 [11-14]. Deletion of synaptotagmin-7 results in glucose intolerance, and impaired insulin secretion [13]. Synaptotagmin-7 knockout (KO) mice maintain normoglycaemia and exhibit normal insulin sensitivity, insulin synthesis, islet architecture and beta cell ultrastructural organisation [13]. They also have lower body weight than their littermate controls [13]. Furthermore, synaptotagmin-7 regulates insulin and glucagon secretion to different extents. While insulin secretion is partially impaired, glucagon secretion is nearly abolished in synaptotagmin-7 $\mathrm{KO}$ mice $[12,13]$.

In the present study we investigated the relative contributions of impaired insulin response and dysregulated glucagon secretion in the development of hyperglycaemia and diabetes. We challenged synaptotagmin-7 KO and control mice with a high-fat diet (HFD) to induce peripheral insulin insensitivity, and measured their insulin and glucagon responses, as well as blood glucose levels. Our results show that dysregulated glucagon secretion plays a previously underappreciated role in promoting hyperglycaemia and the ensuing diabetes.

\section{Methods}

Animal welfare All experiments involving animals were reviewed and approved by the Institutional Animal Care and Use Committee of A*STAR Biomedical Sciences Institutes.

Synaptotagmin-7 KO mice The synaptotagmin-7 KO mice were generated as previously described [15]. All mice used in this study were bred and housed in our animal care facilities. At 12 weeks of age, synaptotagmin-7 KO and control male mice were each divided into two groups and were fed either an HFD or a low-fat diet (LFD, catalogue no. D12451 and D12450B, respectively; Research Diets, New Brunswick, NJ, USA) for 14 weeks. Body weight and resting glucose levels were measured every 1 or 2 weeks.

Blood glucose, insulin and glucagon measurements Blood samples $(4 \mu \mathrm{l})$ for both resting (non-fasted) synaptotagmin$7 \mathrm{KO}$ and control mice were taken from the tail vein every 1 or 2 weeks. Blood glucose concentrations were determined by using an Accu-check advantage glucometer (Roche, Mannheim, Germany). Resting insulin and glucagon levels were measured at 4 and 14 weeks of HFD feeding after $2 \mathrm{~h}$ of fasting (to avoid postprandial insulin increase) by using mouse endocrine multiplex kits (Linco Research, St Charles, MO, USA). Fasting glucose and insulin levels at 4 and 14 weeks of HFD feeding were measured in mice that had been fasted overnight.

Glucose tolerance tests, insulin tolerance tests and in vivo insulin secretion measurements Glucose tolerance tests (GTT), insulin tolerance tests (ITT) and in vivo insulin secretion measurements were performed on synaptotagmin$7 \mathrm{KO}$ and control mice after 14 weeks of HFD or LFD feeding essentially as previously described [13]. Plasma insulin concentrations were determined by Ultrasensitive Mouse Insulin enzyme-linked immunosorbent assay (Mercodia, Uppsala, Sweden). The AUC was calculated by using Interactive Data Language (IDL) based on a fivepoint Newton-Cotes integration formula. For the ITT, data are presented as a percentage of the basal glucose level.

In vivo glucagon secretion measurements Mice were fasted for $2 \mathrm{~h}$ with free access to water before they were weighed and injected i.p. with insulin (Actrapid) at $1 \mathrm{U} / \mathrm{kg}$ of body weight. Blood samples of $\sim 35 \mu$ l were collected from the tail vein for determination of blood glucose and plasma glucagon levels before and 20 and $40 \mathrm{~min}$ after the injection. Blood glucose was determined using an Accu-check advantage glucometer (Roche), and plasma glucagon using a glucagon RIA (Millipore, Billerica, MA, USA) according to the manufacturer's instructions. AUC was calculated by following the trapezoid rule after baseline subtraction.

Islet isolation and glucagon secretion measurements Islets were isolated from synaptotagmin-7 $\mathrm{KO}$ and control mice 
at the end of 14 weeks of HFD feeding by liberase (Roche) digestion, and cultured in RPMI Advanced (Invitrogen, Auckland, New Zealand) supplemented with $10 \%$ heatinactivated fetal bovine serum, $2 \mathrm{mmol} / \mathrm{l}$ L-glutamine, $1 \%$ penicillin-streptomycin and $15 \mathrm{mmol} / \mathrm{l}$ HEPES. All subsequent handling was performed in Krebs-Ringer-HEPES (KRH) medium containing (in mmol/l): $130 \mathrm{NaCl}, 4.7 \mathrm{KCl}$, $1.2 \mathrm{KH}_{2} \mathrm{PO}_{4}, 1.2 \mathrm{MgSO}_{4}, 2.56 \mathrm{CaCl}_{2}, 1 \mathrm{mg} / \mathrm{ml} \mathrm{BSA}$ and $20 \mathrm{mmol} / \mathrm{l}$ HEPES (pH 7.4) supplemented with $10 \mathrm{mmol} /$ 1 glucose. Similar-sized islets from a single mouse (ten islets per batch) were first incubated in $200 \mu \mathrm{l}$ of $\mathrm{KRH}$ buffer containing $1 \mathrm{mmol} / 1$ glucose for $15 \mathrm{~min}$ at $37^{\circ} \mathrm{C}$. The medium was collected and islets were further incubated in $200 \mu \mathrm{l}$ of KRH containing $10 \mathrm{mmol} / \mathrm{l}$ glucose for $15 \mathrm{~min}$. Glucagon concentrations in the two fractions were measured using a Glucagon RIA (Linco Research). Inhibition of glucagon secretion at $10 \mathrm{mmol} / \mathrm{l}$ glucose was expressed as the percentage of stimulated secretion at $1 \mathrm{mmol} / 1$ glucose.

Pyruvate tolerance tests Pyruvate tolerance tests were performed in HFD-fed synaptotagmin-7 $\mathrm{KO}$ and control mice after $24 \mathrm{~h}$ of fasting. The mice were injected i.p. with pyruvate ( $2 \mathrm{~g} / \mathrm{kg}$ body weight), and blood glucose levels were determined before and 30, 60, 90 and $120 \mathrm{~min}$ after injection [16].

Glycogen measurements Liver samples from fasted and non-fasted mice on the LFD and fasted mice on the HFD were snap-frozen in liquid nitrogen. Approximately $50 \mathrm{mg}$ from each sample was boiled in $1 \mathrm{ml}$ of $30 \% \mathrm{KON}$ for $20 \mathrm{~min}$ and centrifuged at $16,000 \mathrm{~g}$ for $10 \mathrm{~min}$. After protein separation, glycogen was precipitated by $100 \%$ ethanol, centrifuged at $16,000 \mathrm{~g}$ for $5 \mathrm{~min}$, washed and dissolved in $100 \mu \mathrm{l}$ of distilled water. Glycogen concentration was measured using a Glycogen Assay Kit (Biovision, Mountain View, CA, USA).

Statistical analysis Data are presented as mean \pm SEM. Statistical comparisons were made by two-tailed Student's $t$ test or by ANOVA followed by Tukey's test. The significance limit was set at $p<0.05$.

\section{Results}

HFD-induced obesity and hyperglycaemia in control mice Synaptotagmin-7 KO mice exhibited glucose intolerance and reduced insulin secretion, along with nearly abolished stimulated glucagon secretion. To determine whether reduced insulin sensitivity on top of impaired insulin secretion would significantly accelerate the progression of hyperglycaemic development in synaptotagmin-7 $\mathrm{KO}$ mice, we challenged KO mice with the HFD and compared the KO group with control mice on the HFD. We first monitored body weight gain in synaptotagmin-7 KO and control mice on the HFD. As we previously reported [13], synaptotagmin-7 KO mice had a lower body weight than their control at the start of HFD feeding. Both groups showed significant weight gain on the HFD $(63 \pm 5$ and $67 \pm$ $5 \%$ over starting weight, $\mathrm{KO}$ vs control, $n=10$ for each genotype), and the weight difference persisted over the 14 weeks of HFD feeding (Fig. 1a). Unexpectedly, the average blood glucose level remained relatively stable in synaptotagmin-7 KO mice despite significant body weight gain over the 14 weeks of HFD feeding (Fig. 1b), while blood glucose levels in control mice started to increase after 5 weeks of HFD feeding, and continued to rise over the next 9 weeks (Fig. 1b). When compared with synaptotagmin-7 KO mice, control mice showed significantly higher blood glucose levels from week 5 to the end of week 14, except for week 7 (Fig. 1b); the level reached $13.2 \pm 1.0 \mathrm{mmol} / \mathrm{l}$ at the end of the 14 weeks of HFD feeding (Fig. 1c). By the end of the 14-week HFD study, more than $60 \%$ of controls (64\%) showed blood glucose levels above $10 \mathrm{mmol} / \mathrm{l}$, a level that is considered hyperglycaemia in humans, whereas in synaptotagmin-7 KO mice the blood glucose level reached $7.2 \pm 0.3 \mathrm{mmol} / 1$ at the end of the 14 weeks of HFD feeding, and none of the synaptotagmin-7 KO mice developed hyperglycaemia
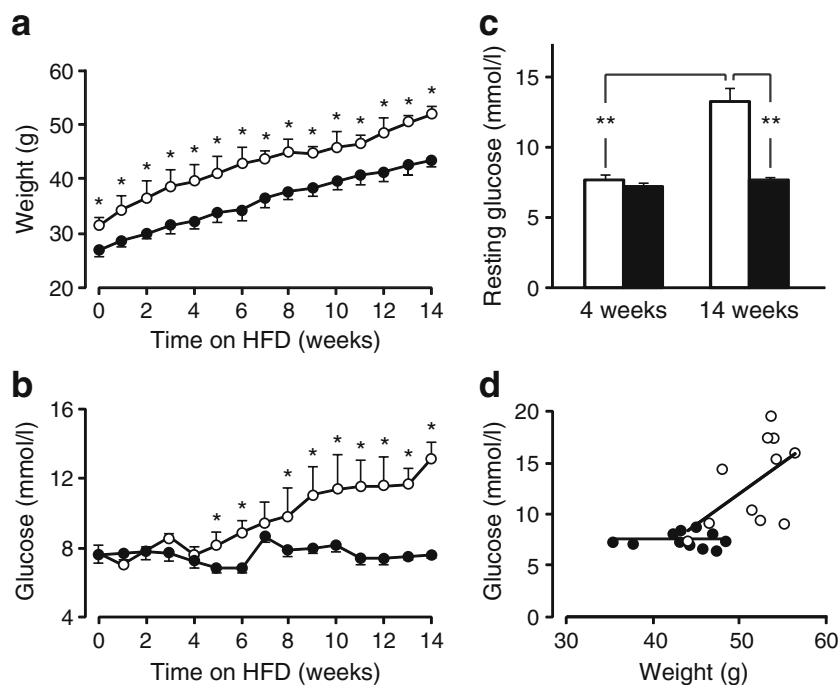

Fig. 1 Synaptotagmin-7 KO mice are protected from developing hyperglycaemia on prolonged HFD feeding. Body weight (a) and glucose levels (b) in synaptotagmin-7 $\mathrm{KO}$ and control mice were measured in the course of 14 weeks of HFD feeding. c Resting glucose levels at the end of 4 weeks and 14 weeks of HFD feeding. d Correlation of body weight and blood glucose levels at the end of 14 weeks of HFD feeding. $y=0.60 x-18.0, r=0.056$ for the control group; $y=0.00 x+7.4, r=0.02$ for the synaptotagmin-7 KO group. Control: white circles $(\mathbf{a}, \mathbf{b}, \mathbf{d})$ or white bars $(\mathbf{c})$; synaptotagmin-7 KO: black circles $(\mathbf{a}, \mathbf{b}, \mathbf{d})$ or black bars $(\mathbf{c})$. Data are mean \pm SEM. ${ }^{*} p<$ $0.05, * * p<0.01, n=11$ for each genotype 
(Fig. 1c). Interestingly, there appeared to be a positive correlation between blood glucose level and body weight in the control group: at the end of the HFD study, mice with higher body weight exhibited higher glucose levels, while no apparent correlation was observed in synaptotagmin-7 KO group (Fig. 1d).

Continued glucose intolerance in synaptotagmin-7 KO mice after HFD feeding Synaptotagmin-7 KO mice on a normal chow diet exhibited normal fasting blood glucose levels, but glucose intolerance during the GTT [13]. We first tested fasting glucose levels after 4 and 14 weeks of HFD feeding in synaptotagmin-7 KO and control mice. Feeding the HFD led to elevated fasting glucose levels in both genotypes when compared with age-matched mice that were fed the LFD (data not shown). However, fasting glucose levels were not different between synaptotagmin-7 $\mathrm{KO}$ and control mice after either 4 weeks ( $6.5 \pm 0.4$ vs $6.5 \pm 0.2 \mathrm{mmol} / \mathrm{l}$, synaptotagmin-7 KO vs control; $n=10$ for each genotype, NS) or 14 weeks of HFD feeding ( $7.5 \pm 0.5$ vs $7.9 \pm 0.7 \mathrm{mmol} / 1$, synaptotagmin- 7 KO vs control; $n=10$ for each genotype, NS).

We then compared glucose tolerance in synaptotagmin-7 $\mathrm{KO}$ and control mice after 14 weeks of HFD feeding. There was no difference in starting glucose levels between the two groups. However, the synaptotagmin-7 KO group exhibited higher blood glucose levels than the control group 30,60 and $90 \mathrm{~min}$ after glucose challenge (Fig. 2a). Furthermore, synaptotagmin-7 KO mice showed higher cumulative blood glucose levels than the control group during the GTT (Fig. 2b). To compare the progression of glucose intolerance in synaptotagmin-7 KO mice on the HFD, mice of the same age that were fed with LFD were also tested. Synaptotagmin-7 KO mice on the HFD showed greater impairment of glucose tolerance than those on the LFD, i.e. even more delayed clearance of glucose, which was reflected as higher glucose levels at $30 \mathrm{~min}$ after glucose challenge (Fig. 2a). In HFD-fed control mice, the impairment of glucose tolerance displayed a similar pattern (Fig. 2). Together, these results demonstrate that after 14 weeks of HFD feeding, control mice became glucoseintolerant, while synaptotagmin-7 KO mice exhibited continued and further worsening of glucose intolerance.

Impaired insulin sensitivity in HFD-fed synaptotagmin-7 $\mathrm{KO}$ and control mice We previously reported glucose intolerance in synaptotagmin-7 KO mice on a normal chow diet and attributed this to impaired insulin secretion, as the mice showed no sign of reduced insulin sensitivity [13]. Prolonged HFD feeding is a well-established method of inducing insulin resistance and diabetes. To test whether insulin sensitivity was affected in synaptotagmin-7 KO and control mice by the HFD, we performed an ITT on these mice after 4 and 14 weeks of HFD feeding. At 4 weeks of HFD
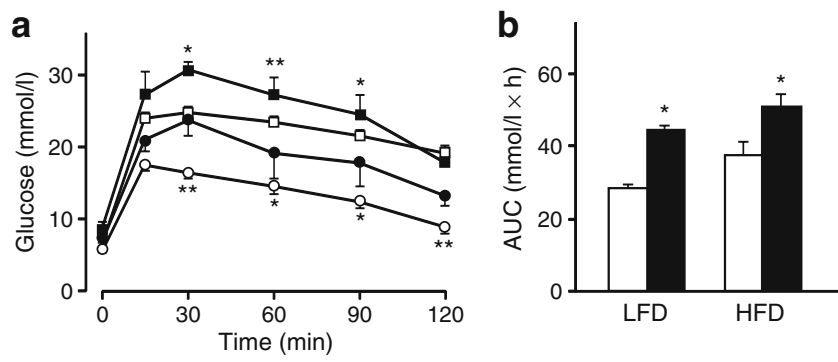

Fig. 2 Continued and progressive worsening of glucose intolerance in synaptotagmin-7 KO mice. a Glucose tolerance tests were performed on synaptotagmin-7 KO and control mice at the end of 14 weeks of HFD or LFD feeding. White circles, LFD-fed control; white squares, LFD-fed synaptotagmin-7 KO; black circles, HFD-fed control; black squares, HFD-fed synaptotagmin-7 KO. b AUC calculated based on data in a. White bars, control; black bars, synaptotagmin-7 KO. Data are mean \pm SEM, $n=6$ for each group. Synaptotagmin-7 KO vs control mice of the same diet group: $* p<0.05,{ }^{* *} p<0.01$

feeding, there was no difference in glucose response after insulin injection between HFD- and LFD-fed synaptotagmin$7 \mathrm{KO}$ mice. However, control mice on the HFD showed slower clearance of blood glucose $20 \mathrm{~min}$ after insulin injection when compared with control mice on the LFD (Fig. 3a). This indicates early impairment of insulin sensitivity in control but not in synaptotagmin-7 KO mice on the HFD. When the ITT was performed at the end of 14 weeks of HFD feeding, control mice on the HFD continued to show impaired insulin sensitivity, while synaptotagmin-7 KO mice developed a similar degree of insensitivity to insulin (Fig. 3b). We also observed lower glucose levels for HFD-fed synaptotagmin-7 KO mice $60 \mathrm{~min}$ after insulin injection at 4 and 14 weeks of HFD feeding compared with the control group (Fig. 3). This is
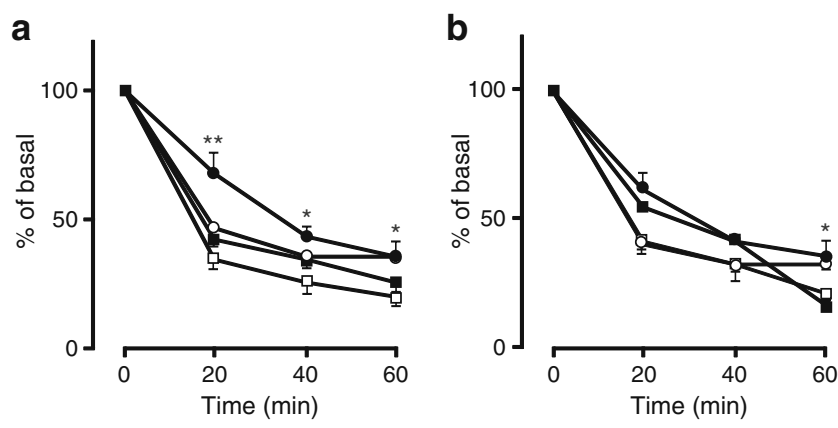

Fig. 3 Development of impaired insulin sensitivity in HFD-fed synaptotagmin-7 $\mathrm{KO}$ and control mice. Insulin tolerance tests were performed in synaptotagmin-7 KO and control mice at the end of 4 weeks (a) or 14 weeks (b) of HFD or LFD feeding. Data are mean \pm SEM. In a, $n=6,9,6$ and 8 for synaptotagmin-7 KO on the HFD (black squares) and LFD (white squares) and for controls on the HFD (black circles) and LFD (white circles), respectively; in $\mathbf{b}, n=5,9,6$ and 8 for synaptotagmin-7 KO on the HFD (black squares) and LFD (white squares) and for controls on the HFD (black circles) and LFD (white circles), respectively. Synaptotagmin-7 KO vs control mice of the same diet group: ${ }^{*} p<0.05,{ }^{* *} p<0.01$ 
probably due to the impaired glucagon secretion in synaptotagmin-7 KO mice, as prolonged hypoglycaemia at this time point is unlikely to be the result of increased insulin sensitivity [12].

Compensatory enhancement of insulin secretion in HFDfed synaptotagmin-7 KO and control mice In the course of type 2 diabetes progression, pancreatic beta cell dysfunction is initially associated with compensatory hyperinsulinaemia, and eventually with diminished stimulated insulin secretion [17]. To assess the effects of HFD feeding on the insulin response and the extent of compensatory hyperinsulinaemia in synaptotagmin-7 KO and control mice, we first measured fasting and resting insulin levels after 4 and 14 weeks of HFD feeding. We reported previously that LFD-fed synaptotagmin-7 KO mice showed lower fasting insulin levels, but similar resting insulin levels when compared with control mice on LFD [13]. Although the HFD led to significantly elevated insulin levels in both synaptotagmin-7 KO and control mice (Fig. 4a), probably as a compensatory mechanism to overcome impaired insulin sensitivity, the same trend persisted after 4 weeks or 14 weeks of HFD feeding: HFD-fed synaptotagmin-7 KO mice continued to show lower fasting insulin levels than HFD-fed control mice (week 4, $0.59 \pm 0.07 \mathrm{vs} 1.22 \pm 0.23 \mathrm{ng} / \mathrm{ml}, n=10$ for each genotype, $p<0.01$; week $14,0.76 \pm 0.17$ vs $1.65 \pm 0.15 \mathrm{ng} / \mathrm{ml}$, $n=10$ for each genotype, $p<0.01$ ), while there was still no difference in resting insulin levels between HFD-fed synaptotagmin-7 $\mathrm{KO}$ and control mice (4 weeks, $1.9 \pm 0.2$ and $3.2 \pm 0.5 \mathrm{ng} / \mathrm{ml}$ for synaptotagmin-7 $\mathrm{KO}$ and control mice, respectively, $n=10$ for each group, NS; 14 weeks, $3.7 \pm 0.7$ and $4.1 \pm 0.6 \mathrm{ng} / \mathrm{ml}$ for synaptotagmin- $7 \mathrm{KO}$ and control mice, respectively; $n=10$ for each group, NS). We
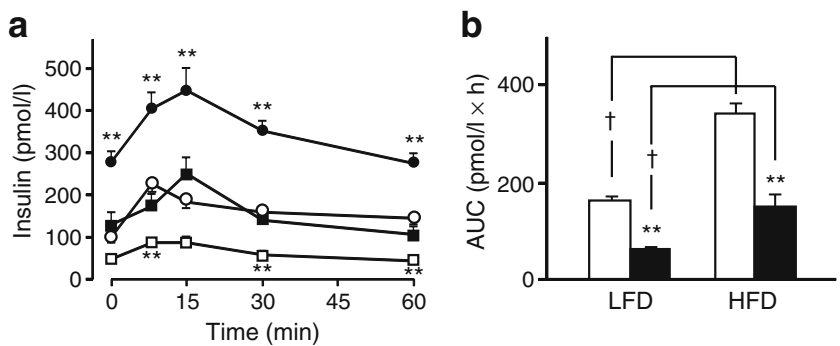

Fig. 4 Compensatory increase in insulin secretion in synaptotagmin-7 $\mathrm{KO}$ and control mice after 14 weeks of HFD feeding. a Acute insulin response to glucose $(2 \mathrm{mg} / \mathrm{g}$ body weight, i.p.) was examined in synaptotagmin-7 KO and control mice after 14 weeks of HFD or LFD feeding. b Glucose-induced insulin secretion calculated by integrating the AUC after baseline subtraction. Data are presented as mean \pm SEM. $n=10,10,10$ and 16 for synaptotagmin-7 KO on the HFD (black squares) and LFD (white squares) and for controls on the HFD (black circles) and LFD (white circles), respectively. In b, control, white bar; synaptotagmin-7 KO, black bar. Synaptotagmin-7 KO vs control mice of the same diet group: ${ }^{* *} p<0.01 .{ }^{\dagger} p<0.01$ for comparisons of LFDand HFD-fed mice with the same genotype also measured the acute insulin response after glucose challenge in synaptotagmin-7 $\mathrm{KO}$ and control mice at 14 weeks of HFD feeding, and compared it with the response in age-matched mice on LFD. Consistent with our previous report, LFD-fed synaptotagmin-7 KO mice showed lower insulin secretion than LFD-fed control mice (Fig. 4) [13]. HFD feeding resulted in a dramatic increase in starting insulin levels, as well as significantly enhanced insulin secretion, in both synaptotagmin-7 KO and control mice (Fig. 4).

Lack of inhibition of glucagon secretion by high glucose in HFD-fed control mice Although less mentioned, like insulin, glucagon also plays a central role in regulating glucose homeostasis. As both synaptotagmin-7 KO and control mice showed similar levels of reduced insulin sensitivity after 14 weeks of HFD feeding (Fig. 3b), only HFD-fed control mice developed hyperglycaemia (Fig. 1c), even though they had significantly higher insulin secretion after glucose challenge than HFD-fed synaptotagmin-7 KO mice (Fig. 4). This paradoxical finding prompted us to investigate the contributions from glucagon. We first measured resting plasma glucagon levels at 4 weeks and 14 weeks of HFD feeding. As synaptotagmin-7 is the principal calcium sensor for glucagon secretion, synaptotagmin-7 KO mice showed significantly reduced glucagon levels [12]. The difference in resting glucagon levels persisted in HFD-fed mice and became greater at the end of 14 weeks of HFD feeding when compared with that at the end of 4 weeks of HFD feeding (Fig. 5a). Furthermore, the resting glucagon level in control mice was nearly doubled at 14 weeks compared with 4 weeks of HFD feeding, while no increase in glucagon level was observed in synaptotagmin-7 KO mice (Fig. 5a). We next examined hypoglycaemia-induced glucagon secretion in vivo in synaptotagmin-7 $\mathrm{KO}$ and control mice at the end of 14 weeks of HFD feeding. Hypoglycaemia stimulated a rapid and significant increase in glucagon secretion in control mice, but only a slight increase in synaptotagmin-7 KO mice (Fig. 5b). Total glucagon secretion, as calculated from the AUC after baseline subtraction, was reduced by more than $80 \%$ in synaptotagmin- $7 \mathrm{KO}$ mice when compared with control mice (Fig. 5c), consistent with the function of synaptotagmin-7 as the principal calcium sensor for glucagon secretion [12]. In normal physiological situations, glucagon secretion is inhibited at high glucose levels. The significantly higher resting glucagon level in the presence of hyperglycaemia in HFD-fed control mice (Figs 1c and 5a) suggested that regulation of glucagon secretion by high glucose was impaired. We tested the intrinsic islet response to high glucose by measuring glucoseinduced inhibition of glucagon secretion in isolated pancreatic islets from synaptotagmin-7 $\mathrm{KO}$ and control mice. Intact islets were consecutively incubated at 1 and $10 \mathrm{mmol} / \mathrm{l}$ 


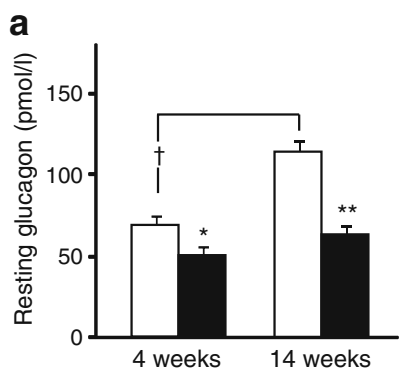

b

C

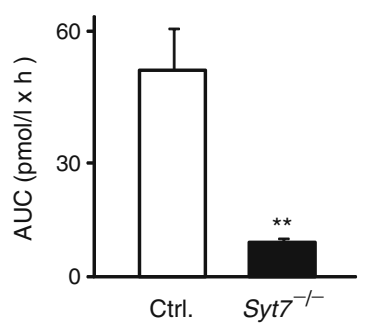

d
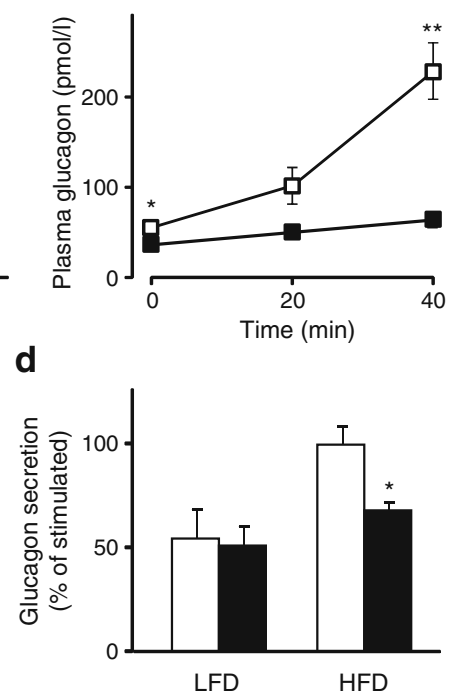

Fig. 5 Lack of inhibition of glucagon secretion by high glucose in isolated islets from HFD-fed control mice. a Resting glucagon levels in synaptotagmin-7 KO (black bar) and control (white bar) mice at the end of 4 and 14 weeks of HFD feeding. $n=11$ for each group. Synaptotagmin-7 KO vs control mice: ${ }^{*} p<0.05,{ }^{*} p<0.01 .{ }^{\dagger} p<0.01$ for comparison of control mice after 4 weeks on the HFD and 14 weeks on the HFD. b In vivo glucagon secretion was measured prior to and 20 and $40 \mathrm{~min}$ after i.p. injection of insulin in synaptotagmin-7 KO (black squares) and control (white squares) mice after 14 weeks on the HFD. $n=7$ for each group, ${ }^{*} p<0.05, * * p<0.01$. c Hypoglycaemia-stimulated glucagon secretion was calculated as the AUC after baseline subtraction based on data in b. $n=7$ for each group, ${ }^{* *} p<0.01$. d Suppression of glucagon secretion by high glucose was examined in isolated islets from synaptotagmin-7 KO (black bars) and control (white bars) mice after 14 weeks of HFD feeding. $n=12,12,13$ and 12 for synaptotagmin-7 KO on the HFD and LFD and controls on the HFD and LFD, respectively. ${ }^{*} p<0.05$

glucose. High glucose $(10 \mathrm{mmol} / \mathrm{l})$ inhibited glucagon secretion to about half of the level measured at low glucose $(1 \mathrm{mmol} / \mathrm{l})$ in isolated islets from LFD-fed synaptotagmin-7 $\mathrm{KO}$ and control mice (Fig. 5d). For islets isolated from HFDfed mice, high glucose-induced inhibition of glucagon secretion was maintained in synaptotagmin-7 KO mice, but completely absent in control mice, indicating severe dysregulation of glucagon release in control but not in synaptotagmin-7 KO mice (Fig. 5d).

Increased hepatic glucose production in HFD-fed control mice As synaptotagmin-7 KO mice had significantly lower stimulated glucagon secretion than control mice, we expected a lower fasting blood glucose level in the $\mathrm{KO}$ mice. However, there was no difference in glucose levels between synaptotagmin-7 KO and control mice after overnight fasting (see above). Stimulated glucagon secretion is triggered at low glucose levels, such as after extended fasting. We tested this notion by measuring blood glucose levels of synaptotagmin-7 and control mice after
$24 \mathrm{~h}$ of fasting. Indeed, synaptotagmin-7 $\mathrm{KO}$ mice exhibited lower blood glucose levels than their control mice $(6.3 \pm 0.5$ vs $7.8 \pm 0.4 \mathrm{mmol} / 1, n=7$ for each genotype, $p<0.05$ ). To evaluate hepatic glucose production, we measured glucose levels in response to injection of the gluconeogenic substrate pyruvate in HFD-fed synaptotagmin-7 KO and control mice. Injection of pyruvate led to significantly higher blood glucose levels in both groups of mice, but HFD-fed control mice displayed a greater increase in glucose levels than HFD-fed synaptotagmin-7 KO mice (Fig. 6), suggesting higher gluconeogenesis in the control mice. We also measured liver glycogen levels in HFD-fed mice. Control mice exhibited a significantly lower glycogen level than synaptotagmin- $7 \mathrm{KO}$ mice $(9.6 \pm 0.8$ vs $14.6 \pm 2.1 \mathrm{mmol} / 1, n=$ 6 for each genotype, $p<0.05)$, consistent with increased glycogenolysis as a consequence of the higher glucagon level. Together, these results demonstrate increased hepatic glucose production in control mice when compared with synaptotagmin-7 KO mice.

\section{Discussion}

Type 2 diabetes develops when pancreatic beta cells fail to secrete sufficient insulin to meet peripheral insulin demands [4]. Because of the beta cell- or insulin-centric view of the disease, overwhelmingly more efforts have been devoted to the study of beta cell functions and dysfunctions and of insulin secretion regulation and failure in normal physiology and during disease progression than to the study of pancreatic alpha cells and glucagon [11]. Glucagon is closely involved in the control of glucose levels by regulating glucose production in the liver, and forms a yin-yang partnership with insulin in the regulation of glucose homeostasis. Although increased glucagon secretion is commonly de-
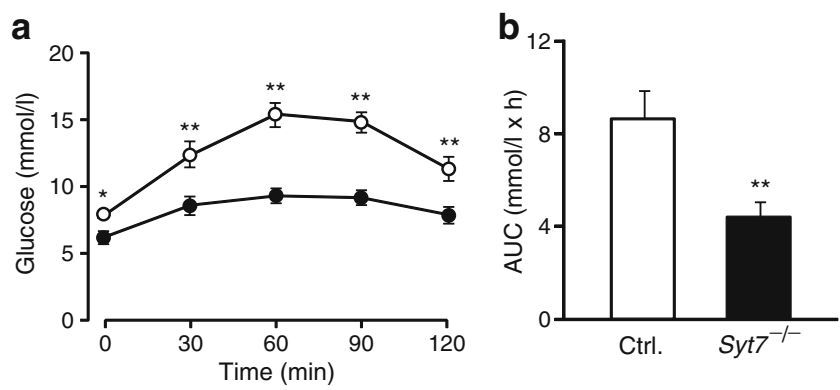

Fig. 6 Increased hepatic glucose production in HFD-fed control mice. a Blood glucose levels were determined in HFD-fed synaptotagmin-7 $\mathrm{KO}$ (black circles) and control (white circles) mice in pyruvate tolerance tests. b Cumulative hepatic glucose production by gluconeogenesis was calculated as the AUC based on data in a. $n=8$ for synaptotagmin-7 KO mice and $n=7$ for control mice. Data are mean \pm SEM. ${ }^{*} p<0.05, * * p<0.01$ 
scribed in diabetes, it is rarely mentioned in prediabetic conditions [1, 2, 8, 9]. Moreover, elevated glucagon secretion is usually referred to as a consequence of diabetes, and an active role of glucagon in promoting hyperglycaemia remains controversial or ignored $[2,9,10]$.

An alternative to the insulin-centric view in diabetes development is the bi-hormonal abnormality hypothesis proposed by Unger and Orci in 1975 [10]. This model states that the major consequence of absolute or relative insulin deficiency is glucose underutilisation, and that absolute or relative glucagon excess is the principal factor in the overproduction of glucose in diabetes [10]. In support of the bi-hormonal model, a number of studies have demonstrated that pancreatic alpha cells are hyporesponsive to glucose-induced suppression and hyperresponsive to the stimulatory effects of amino acids in type 2 diabetes, although these defects in alpha cell function could also be considered as secondary to defective glucose sensing in beta cells (see review and references cited in [2]).

Synaptotagmin-7 KO mice show impaired insulin secretion and glucose intolerance [13], so we expected they would develop hyperglycaemia much sooner when combined with HFD-induced insulin insensitivity. Unexpectedly, however, defective insulin secretion and reduced insulin sensitivity were not sufficient to cause hyperglycaemia in the absence of enhanced glucagon secretion. The HFD-fed control mice, which showed an extent of reduced insulin sensitivity similar to that seen in the HFD-fed synaptotagmin-7 KO mice, but significantly elevated insulin secretion, developed resting hyperglycaemia, with a concurrent increase and dysregulation in glucagon secretion. HFD-fed mice of both genotypes showed delayed glucose clearance, indicating progressive beta cell dysfunction [17]. Accordingly, both synaptotagmin$7 \mathrm{KO}$ and control mice became more glucose-intolerant. Why did the control mice, but not the synaptotagmin-7 KO mice, develop hyperglycaemia even though their beta cell dysfunction and glucose intolerance were less severe than in synaptotagmin-7 KO mice? There are two likely possibilities: first, synaptotagmin-7 KO mice developed insulin resistance at a slower pace than the control mice, and thus the onset of hyperglycaemia was delayed. We evaluated insulin sensitivity by an ITT and found that synaptotagmin-7 KO mice had higher insulin sensitivity at the end of 4 weeks of HFD feeding (Fig. 3a), although the difference in insulin sensitivity disappeared at the end of 14 weeks of HFD feeding (Fig. 3b). The ITT is a commonly used method of assessing insulin sensitivity; however, the gold standard method is hyperinsulinaemic euglycaemic clamping. Future studies are needed to determine to what extent delayed insulin resistance contributes to protecting synaptotagmin-7 KO mice from developing hyperglycaemia. Second, synaptotagmin-7 KO mice continued to maintain their low glucagon level and glucose inhibition of glucagon secretion even after prolonged HFD feeding, while the HFD-fed control mice exhibited a significantly higher glucagon level and loss of glucose inhibition of glucagon secretion, and the elevated glucagon level led to increased hepatic glucose production (Fig. 6) and the consequent hyperglycaemia.

Glucagon secretion regulation is highly complex. Besides the intrinsic control mechanisms in the regulation of glucagon production, secretory granule biogenesis and glucagon granule exocytosis, external factors, such as paracrine factors from neighbouring beta and delta cells, and neuroendocrine factors also play major roles in the regulation of glucagon secretion [1, 18]. Insulin [19], along with substances released together with insulin, such as $\gamma$ aminobutyric acid [20, 21], islet amyloid polypeptide [22], $\mathrm{Zn}^{2+}$ [23] and ATP [24], suppresses the function of alpha cells (for review, see $[1,25]$ ). One hypothesis regarding glucose inhibition of glucagon secretion is that such action is mediated through insulin or its co-released factors [26, 27]. Interestingly, glucose-induced insulin secretion in synaptotagmin-7 KO mice is impaired [13], which should result in less pronounced inhibition of glucagon. Instead, these mice demonstrate adequate suppression of glucagon release in the presence of high glucose, indicating that inhibition of glucagon secretion by high glucose is also mediated by other factors. Further studies on the regulation of glucagon secretion in synaptotagmin-7 KO mice may aid in understanding how high glucose inhibits glucagon secretion, and in providing valuable insights into mechanisms of dysregulated glucagon secretion in diabetes.

Dysregulation of glucagon secretion is linked to dysfunction of beta cells, but the extent to which insulin secretion must be impaired to cause such an effect is unknown. Therefore, it is possible that dysregulation of glucagon secretion is present at even early stages of beta cell dysfunction, long before diabetes is diagnosed.

In summary, our data show that combined beta cell dysfunction and insulin insensitivity may not be sufficient to induce hyperglycaemia without dysregulated glucagon secretion, and thus reveal a previously underappreciated role of the regulation of glucagon secretion in the development of diabetes. Increased glucagon secretion does not merely aggravate hyperglycaemia at late stages of diabetes, but also actively promotes diabetes development at early stages of diabetes pathogenesis. Our study thus suggests that glucagon control may be an important therapeutic strategy for the management of hyperglycaemia and diabetes.

Acknowledgements We thank C. Li for discussions and advice and Y. Liu, J. Kusunoki, G. Smith and P. Shepherd for help with hepatic glucose production measurements. This study was supported by intramural funding from A*STAR (Agency for Science, Technology and Research, Singapore, Republic of Singapore) Biomedical Research Council (W. Han). 
Duality of interest The authors declare that there is no duality of interest associated with this manuscript.

\section{References}

1. Gromada J, Franklin I, Wollheim CB (2007) Alpha-cells of the endocrine pancreas: 35 years of research but the enigma remains. Endocr Rev 28:84-116

2. Dunning BE, Gerich JE (2007) The role of alpha-cell dysregulation in fasting and postprandial hyperglycemia in type 2 diabetes and therapeutic implications. Endocr Rev 28:253-283

3. Guillausseau PJ, Meas T, Virally M, Laloi-Michelin M, Medeau V, Kevorkian JP (2008) Abnormalities in insulin secretion in type 2 diabetes mellitus. Diabetes Metab 34(Suppl 2):S43-48

4. Ashcroft FM, Rorsman P (2004) Molecular defects in insulin secretion in type-2 diabetes. Rev Endocr Metab Disord 5:135-142

5. Porte DJ, Kahn SE (2001) Beta-cell dysfunction and failure in type 2 diabetes: potential mechanisms. Diabetes 50(Suppl 1):S160-163

6. Porte D Jr (2001) Clinical importance of insulin secretion and its interaction with insulin resistance in the treatment of type 2 diabetes mellitus and its complications. Diabetes Metab Res Rev 17:181-188

7. Virally M, Blickle JF, Girard J, Halimi S, Simon D, Guillausseau PJ (2007) Type 2 diabetes mellitus: epidemiology, pathophysiology, unmet needs and therapeutical perspectives. Diabetes Metab 33:231-244

8. Gromada J, Duttaroy A, Rorsman P (2009) The insulin receptor talks to glucagon? Cell Metab 9:303-305

9. Burcelin R, Knauf C, Cani PD (2008) Pancreatic alpha-cell dysfunction in diabetes. Diabetes Metab 34(Suppl 2):S49-55

10. Unger RH, Orci L (1975) The essential role of glucagon in the pathogenesis of diabetes mellitus. Lancet 1:14-16

11. Gustavsson N, Han W (2009) Calcium-sensing beyond neurotransmitters: functions of synaptotagmins in neuroendocrine and endocrine secretion. Biosci Rep 29:245-259

12. Gustavsson N, Wei SH, Hoang DN et al (2009) Synaptotagmin-7 is a principal $\mathrm{Ca} 2+$ sensor for $\mathrm{Ca} 2+$-induced glucagon exocytosis in pancreas. J Physiol 587:1169-1178

13. Gustavsson N, Lao Y, Maximov A et al (2008) Impaired insulin secretion and glucose intolerance in synaptotagmin-7 null mutant mice. Proc Natl Acad Sci U S A 105:3992-3997
14. Gauthier BR, Wollheim CB (2008) Synaptotagmins bind calcium to release insulin. Am J Physiol Endocrinol Metab 295:E12791286

15. Maximov A, Lao Y, Li $\mathrm{H}$ et al (2008) Genetic analysis of synaptotagmin-7 function in synaptic vesicle exocytosis. Proc Natl Acad Sci U S A 105:3986-3991

16. Miyake K, Ogawa W, Matsumoto M, Nakamura T, Sakaue H, Kasuga M (2002) Hyperinsulinemia, glucose intolerance, and dyslipidemia induced by acute inhibition of phosphoinositide 3kinase signaling in the liver. J Clin Invest 110:1483-1491

17. Weir GC, Bonner-Weir S (2004) Five stages of evolving beta-cell dysfunction during progression to diabetes. Diabetes 53(Suppl 3): S16-21

18. Luft R, Efendic S, Hokfelt T (1978) Somatostatin-both hormone and neurotransmitter? Diabetologia 14:1-13

19. Unger RH (1985) Glucagon physiology and pathophysiology in the light of new advances. Diabetologia 28:574-578

20. Rorsman P, Berggren PO, Bokvist K et al (1989) Glucoseinhibition of glucagon secretion involves activation of GABAAreceptor chloride channels. Nature 341:233-236

21. Wendt A, Birnir B, Buschard K et al (2004) Glucose inhibition of glucagon secretion from rat alpha-cells is mediated by GABA released from neighboring beta-cells. Diabetes 53:1038-1045

22. Panagiotidis G, Salehi AA, Westermark P, Lundquist I (1992) Homologous islet amyloid polypeptide: effects on plasma levels of glucagon, insulin and glucose in the mouse. Diabetes Res Clin Pract 18:167-171

23. Ishihara H, Maechler P, Gjinovci A, Herrera PL, Wollheim CB (2003) Islet beta-cell secretion determines glucagon release from neighbouring alpha-cells. Nat Cell Biol 5:330-335

24. Tuduri E, Filiputti E, Carneiro EM, Quesada I (2008) Inhibition of $\mathrm{Ca} 2+$ signaling and glucagon secretion in mouse pancreatic alphacells by extracellular ATP and purinergic receptors. Am J Physiol Endocrinol Metab 294:E952-960

25. Rorsman P, Salehi SA, Abdulkader F, Braun M, MacDonald PE (2008) K(ATP)-channels and glucose-regulated glucagon secretion. Trends Endocrinol Metab 19:277-284

26. Maruyama H, Hisatomi A, Orci L, Grodsky GM, Unger RH (1984) Insulin within islets is a physiologic glucagon release inhibitor. J Clin Invest 74:2296-2299

27. Franklin I, Gromada J, Gjinovci A, Theander S, Wollheim CB (2005) Beta-cell secretory products activate alpha-cell ATP-dependent potassium channels to inhibit glucagon release. Diabetes 54:18081815 